\title{
High-Field Hole Transport in Strained Si and SiGe by Monte Carlo Simulation: Full Band Versus Analytic Band Models
}

\author{
F. M. BUFLER*, P. GRAF** and B. MEINERZHAGEN \\ Institut für Theoretische Elektrotechnik und Mikroelektronik, FB 1, Postfach 3304 40, \\ Universität Bremen, D-28334 Bremen, Germany
}

\begin{abstract}
Monte Carlo results are presented for the velocity-field characteristics of holes in (i) unstrained $\mathrm{Si}$, (ii) strained $\mathrm{Si}$ and (iii) strained SiGe using a full band model as well as an analytic nonparabolic and anisotropic band structure description. The full band Monte Carlo simulations show a strong enhancement of the drift velocity in strained Si up to intermediate fields, but yield the same saturation velocity as in unstrained $\mathrm{Si}$. The drift velocity in strained $\mathrm{SiGe}$ is also significantly enhanced for low fields while being substantially reduced in the high-field regime. The results of the analytic band models agree well with the full band results up to medium field strengths and only the saturation velocity is significantly underestimated.
\end{abstract}

Keywords: Monte Carlo simulation, analytic and full band structures, strained $\mathrm{Si}$ and $\mathrm{SiGe}$

\section{INTRODUCTION}

The progress in epitaxial growth techniques of unstrained and strained SiGe layers have led to intensified efforts to explore the potential performance enhancements in $\mathrm{SiGe}$ based devices. In particular, the practical usefulness of $p$-MOSFETs with a channel consisting of strained $\mathrm{Si}$ [1] or strained SiGe [2] has been recently demonstrated. Since field effect devices operate in the low-field and in the high-field regime, reliable modeling of hole transport is important for both cases. How- ever, previous publications on hole transport in strained $\mathrm{Si}$ and $\mathrm{SiGe}$ covered only the low field regime [3] or were restricted to strained $\mathrm{SiGe}$ and electric field strengths below $20 \mathrm{kV} / \mathrm{cm}$ [4]. Hence, there is a clear need for investigations of high-field effects like velocity saturation where the consideration of the full band structure is often necessary for accurate results. On the other hand, for devices with realistic Germanium profiles full band Monte Carlo simulations still involve an unmanageable computational burden (e.g. prohibitive memory requirements) and analytic band structure

\footnotetext{
* Corresponding author. Present address: Institut für Integrierte Systeme, Gloridstrasse 35, CH-8092 Zürich, Switzerland.

**Present address: Intermetall, Hans-Bunte-Str. 19, D-79108 Freiburg, Germany.
} 
approximations have to be used instead. The aim of this paper is therefore twofold: On one hand, we perform for the first time full band Monte Carlo simulations for strained $\mathrm{Si}$ and $\mathrm{SiGe}$ in the high-field regime. On the other hand, we present a simple analytic hole band model and evaluate its range of validity.

\section{MODEL DESCRIPTION}

The full band model for strained $\mathrm{Si}$ or $\mathrm{SiGe}$ is obtained by nonlocal empirical pseudopotential calculations including spin-orbit interaction [9]. For the analytic band structure we neglect the warping of the three valence bands $v=1,2,3$ and use a simple parametrization according to

$$
E\left(1+\alpha_{v} E\right)=\frac{\hbar^{2}}{2}\left(\frac{k_{x}^{2}}{m_{\|, v}}+\frac{k_{y}^{2}}{m_{\|, v}}+\frac{k_{z}^{2}}{m_{\perp, v}}\right)
$$

with $E=\varepsilon-\varepsilon_{0, v}$ because of the feasibility of this formula for applications. The scattering mechanisms included are optical phonons and acoustic phonons in the isotropic and elastic equipartition approximation. In SiGe both Si-type and Ge-type phonons are considered and alloy scattering is taken into account with the alloy scattering potential adjusted to drift mobility measurements in unstrained SiGe [10]. Exactly the same coupling constants are used with the full band and the analytic band model. The parameters $\alpha_{v}, m_{\|, v}$ and $m_{\perp, v}$ are adapted to the full band structure for the purpose of transport applications. The starting point is therefore the expression for the Ohmic drift mobility which involves for the scattering processes used only the Density Of States (DOS) and the square of the group velocity averaged over an energy surface $\mathcal{V}^{2}$ [11]. Then a parabolic expression is used for each band to determine the masses $m_{\text {DOS }}$ and $m_{\text {cond }}$ by adjusting the DOS and $\mathcal{V}^{2}$, respectively, up to about $40 \mathrm{meV}$ above the band edge to the respective full band results. For a good transport description the mobility of the analytic band model in Eq. (1) must equal the mobility obtained with the parabolic fits to the full band model. This condition yields for $\alpha=0$ in the unstrained case $\left(m_{\|}=m_{\perp} \equiv m\right)$

$$
m=m_{\text {cond }}^{2 / 5} m_{\text {DOS }}^{3 / 5}
$$

Finally, using this mass $m$ the nonparabolicity factor $\alpha$ is obtained from fitting the DOS up to $1 \mathrm{eV}$. A similar procedure applies in the strained case.

\section{VERIFICATION}

In Figures 1, 2, 3 and 4 drift mobilities and drift velocities resulting from the full band and the analytic band model are compared with experimental data in the case of unstrained $\mathrm{Si}$ and $\mathrm{Ge}$ because an accurate reproduction of the experiments is essential in view of the increased importance of details of phonon and band models in the strained case. Overall good agreement is achieved. Especially the full band model in Figure 2 reproduces accurately the anisotropy of the velocity-field characteristics as well as the saturation drift velocity of Ref. [7]. Within the isotropic band approximation (unstrained case) also the

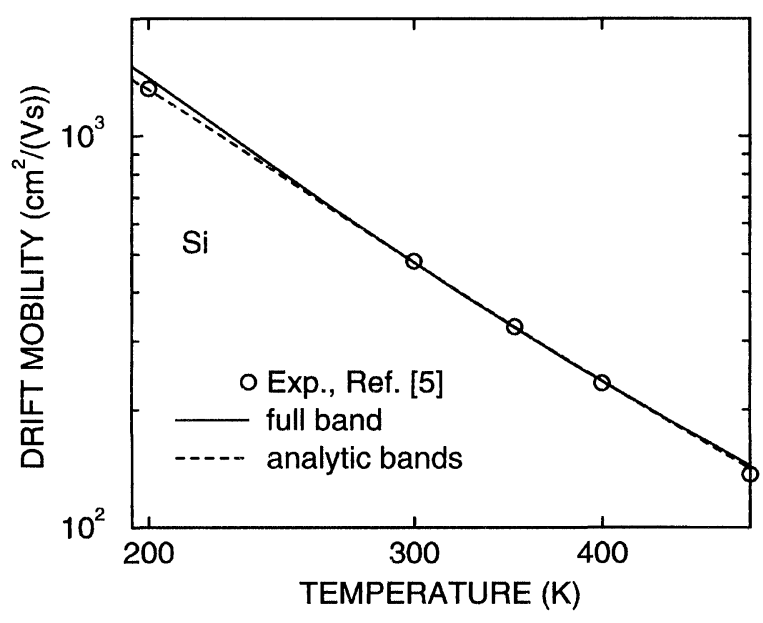

FIGURE 1 Temperature dependence of Ohmic drift mobility for holes in unstrained $\mathrm{Si}$ : comparison of full band model, analytic band model and experimental results. 


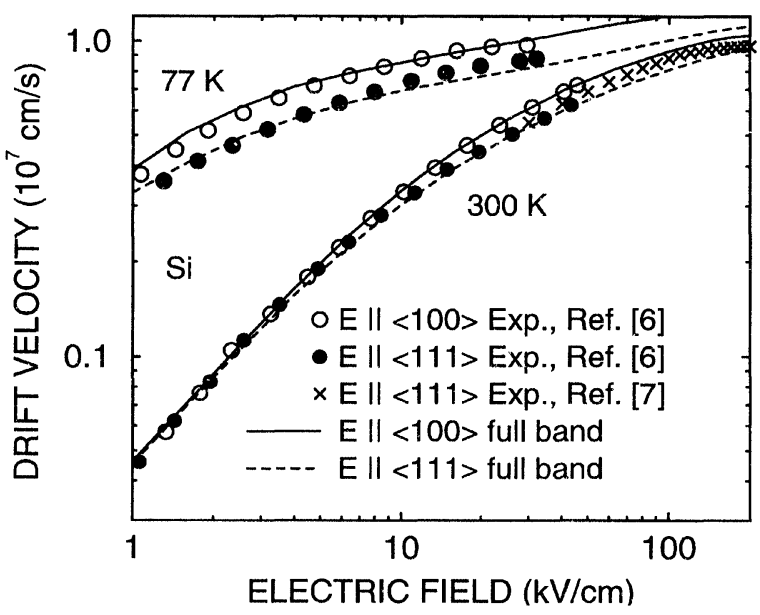

FIGURE 2 Velocity-field characteristics of the full band model for unstrained $\mathrm{Si}$ at 77 and $300 \mathrm{~K}$ in comparison with experimental results.

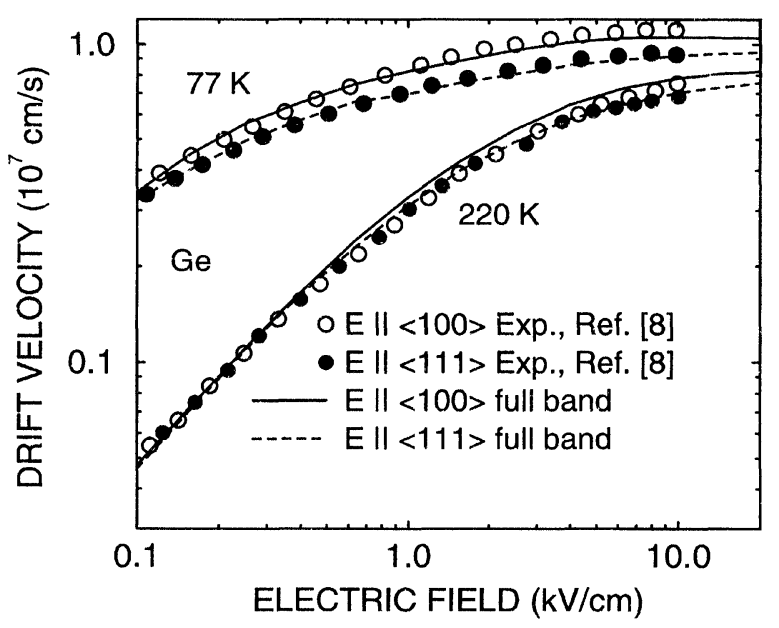

FIGURE 3 Velocity-field characteristics of the full band model for unstrained $\mathrm{Ge}$ at 77 and $220 \mathrm{~K}$ in comparison with experimental results.

analytic band model yields surprisingly good results and only significantly underestimates the drift velocity above $50 \mathrm{kV} / \mathrm{cm}$.

\section{RESULTS}

In Figures 5 and 6 the high-field results for strained $\mathrm{Si}$ and $\mathrm{SiGe}$ are shown. While the value of the saturation drift velocity in unstrained $\mathrm{Si}$ is

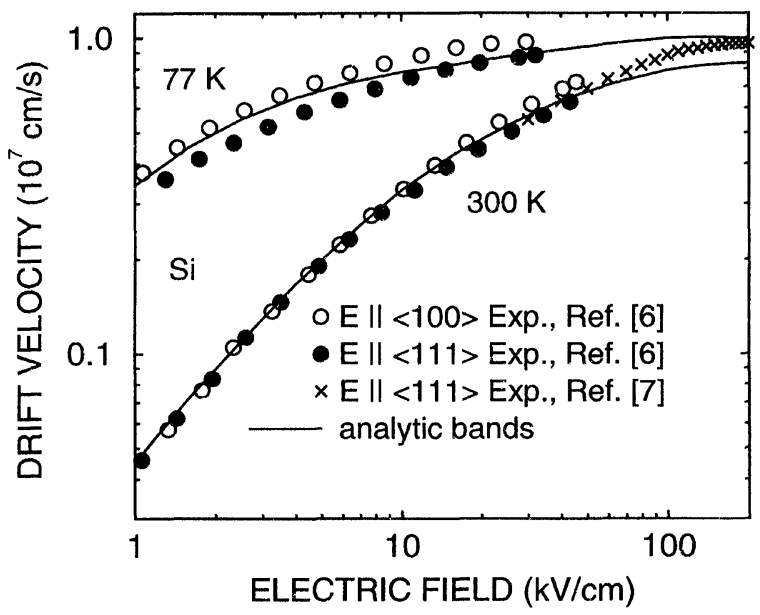

FIGURE 4 Velocity-field characteristics of the analytic band model for unstrained $\mathrm{Si}$ at 77 and $300 \mathrm{~K}$ in comparison with experimental results.

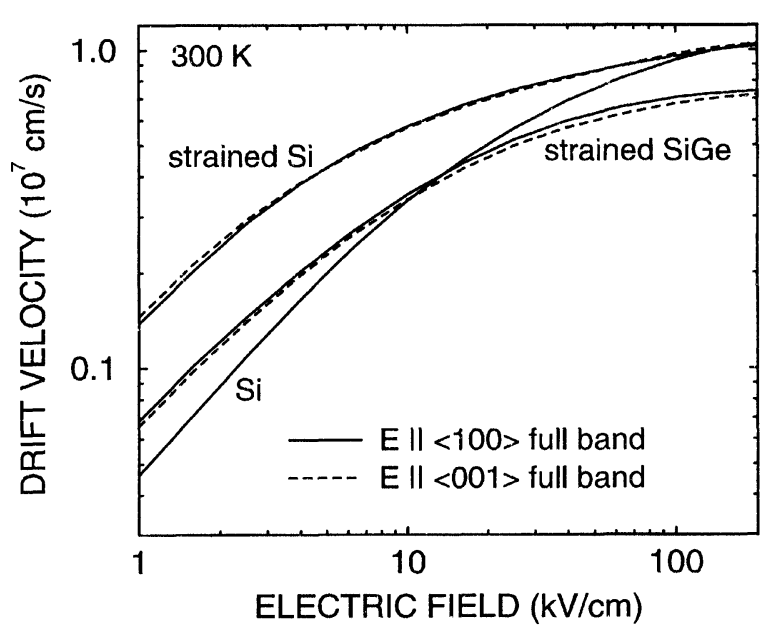

FIGURE 5 Results of the full band model for the velocityfield characteristics at $300 \mathrm{~K}$ in strained $\mathrm{Si}$ grown on a $\mathrm{Si}_{0.7} \mathrm{Ge}_{0.3}$ substrate, in unstrained $\mathrm{Si}$ and in strained $\mathrm{Si}_{0.6} \mathrm{Ge}_{0.4}$ grown on a Si substrate.

retained in strained $\mathrm{Si}$, the drift velocity at lower fields is considerably improved due to the enhanced population of the light hole band. In contrast, the saturation velocity is reduced in strained $\mathrm{SiGe}$, but there is still a substantial improvement up to intermediate fields. But please keep in mind that no realistic estimate of the corresponding device performance can be based on 


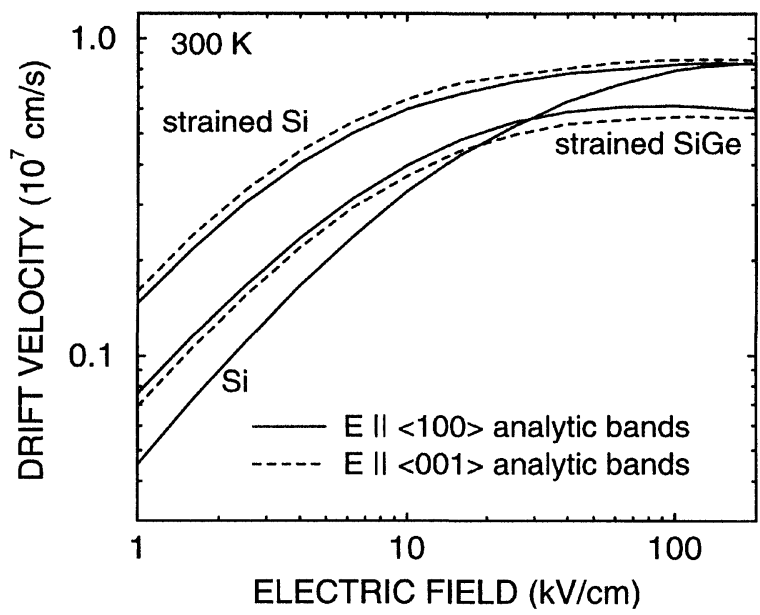

FIGURE 6 Results of the analytic band model for the velocity-field characteristics at $300 \mathrm{~K}$ in strained $\mathrm{Si}$ grown on a $\mathrm{Si}_{0.7} \mathrm{Ge}_{0.3}$ substrate, in unstrained $\mathrm{Si}$ and in strained $\mathrm{Si}_{0.6} \mathrm{Ge}_{0.4}$ grown on a Si substrate.

Figure 5 alone because advantages like the possibility of modulation doping have to be considered for this purpose as well. The analytic band model again underestimates the drift velocity above $50 \mathrm{kV} / \mathrm{cm}$ and somewhat overestimates anisotropy.

\section{Acknowledgements}

This work was supported in part by the Bundesministerium für Bildung, Wissenschaft, Forschung und Technologie under contract no. $01 \mathrm{M} 2416 \mathrm{~A}$. The authors would like to thank P. Vogl (TU München) for discussions, M. M. Rieger (University of Cambridge, U.K.) for calculating the full band structures as well as $\mathrm{K}$. Tittelbach-Helmrich and P. Gaworzewski (Frankfurt/Oder) for providing their experimental mobility data prior to publication.

\section{References}

[1] Nayak, D. K., Goto, K., Yutani, A., Murota, J. and Shiraki, Y. (1996). "High-mobility strained-Si PMOSFET's", IEEE Trans. Electron Devices, 43, 1709-1716.

[2] Risch, L., Fischer, H., Hofmann, F., Schäfer, F., Eller, M. and Aeugle, T. "Fabrication and electrical characterization of Si/SiGe $p$-channel MOSFETs with a delta doped boron layer", in Proc. ESSDERC, Bologna, 1996, 26, $465-468$.

[3] Fischetti, M. V. and Laux, S. E. (1996). "Band structure, deformation potentials, and carrier mobility in strained $\mathrm{Si}$, Ge and SiGe alloys", J. Appl. Phys., 80, 2234-2252.

[4] Hinckley, J. M. and Singh, J. (1990). "Hole transport theory in pseudomorphic $\mathrm{Si}_{1-x} \mathrm{Ge}_{x}$ alloys grown on Si(001) substrates", Phys. Rev. B., 41, 2912-2926.

[5] Green, M. A. (1990). "Intrinsic concentration, effective densities of states, and effective mass in silicon", J. Appl. Phys., 67, 2944-2954.

[6] Canali, C., Ottaviani, G. and Alberigi-Quaranta, A. (1971). "Drift velocity of electrons and holes and associated anisotropic effects in silicon", J. Phys. Chem. Solids, 32, 1707-1720.

[7] Smith, P. M. and Frey, J. (1981). "High-field transport of holes in silicon", Appl. Phys. Lett., 39, 332-333.

[8] Reggiani, L., Canali, C., Nava, F. and Ottaviani, G. (1977). "Hole drift velocity in germanium", Phys. Rev. B., 16, $2781-2791$.

[9] Rieger, M. M. and Vogl, P. (1993). "Electronic-band parameters in strained $\mathrm{Si}_{1-x} \mathrm{Ge}_{x}$ alloys on $\mathrm{Si}_{1-y} \mathrm{Ge}_{y}$ substrates", Phys. Rev. B., 48, 14276-14287.

[10] Tittelbach-Helmrich, K. and Gaworzewski, P. (1997) Unpublished.

[11] Fu, Y., Grahn, K. J. and Willander, M. (1994). "Valence band structure of $\mathrm{Ge}_{x} \mathrm{Si}_{1-x}$ for hole transport calculation”, IEEE Trans. Electron Devices, 41, 26-31.

\section{Authors’ Biographies}

Fabian M. Bufler studied physics at the TU Braunschweig and RWTH Aachen (both Germany) including an academic year at the Université de Grenoble I (France) with a scholarship of the Studienstiftung des deutschen Volkes and received the Dipl.-Phys. degree in 1992. Since then he was working as a research assistant at the Institut für Theoretische Elektrotechnik, RWTH Aachen, and moved in 1995 together with the group of Prof. Bernd Meinerzhagen to the Institut für Theoretische Elektrotechnik und Mikroelektronik, Universität Bremen (Germany). His current research interests are concerned with transport theory and full band Monte Carlo simulation for $\mathrm{SiGe}$ based devices.

Peter Graf received the Diploma in physics from the RWTH Aachen (Technical University of Aachen), Germany, in 1991. At the Institut für Theoretische Elektrotechnik, RWTH Aachen, he then worked on the Monte-Carlo simulation of homogeneous systems and its benefits for hydrodynamic charge transport parameters. After join- 
ing the Institut für Theoretische Elektrotechnik und Mikroelektronik, Universität Bremen, he dealt with algorithms for Monte-Carlo device simulation and their implementation into the institute's Monte-Carlo heterojunction device simulator.

Bernd Meinerzhagen received the Dipl.-Ing. degree in electrical engineering in 1977, the Dipl.Math. degree in mathematics in 1981, the Dr.-Ing. degree in electrical engineering in 1985 and the "venia legendi" in 1995 all from the RWTH Aachen (Germany). During 1986 he was a Member of Technical Staff at AT\&T Bell Laboratories in Allentown (USA) and, after returning to the RWTH Aachen in 1987, became head of the silicon technology modeling and simulation (TCAD) group. In 1995 he was appointed Professor at the University of Bremen, where his current research interests include TCAD and the theory of electromagnetic fields and networks. 

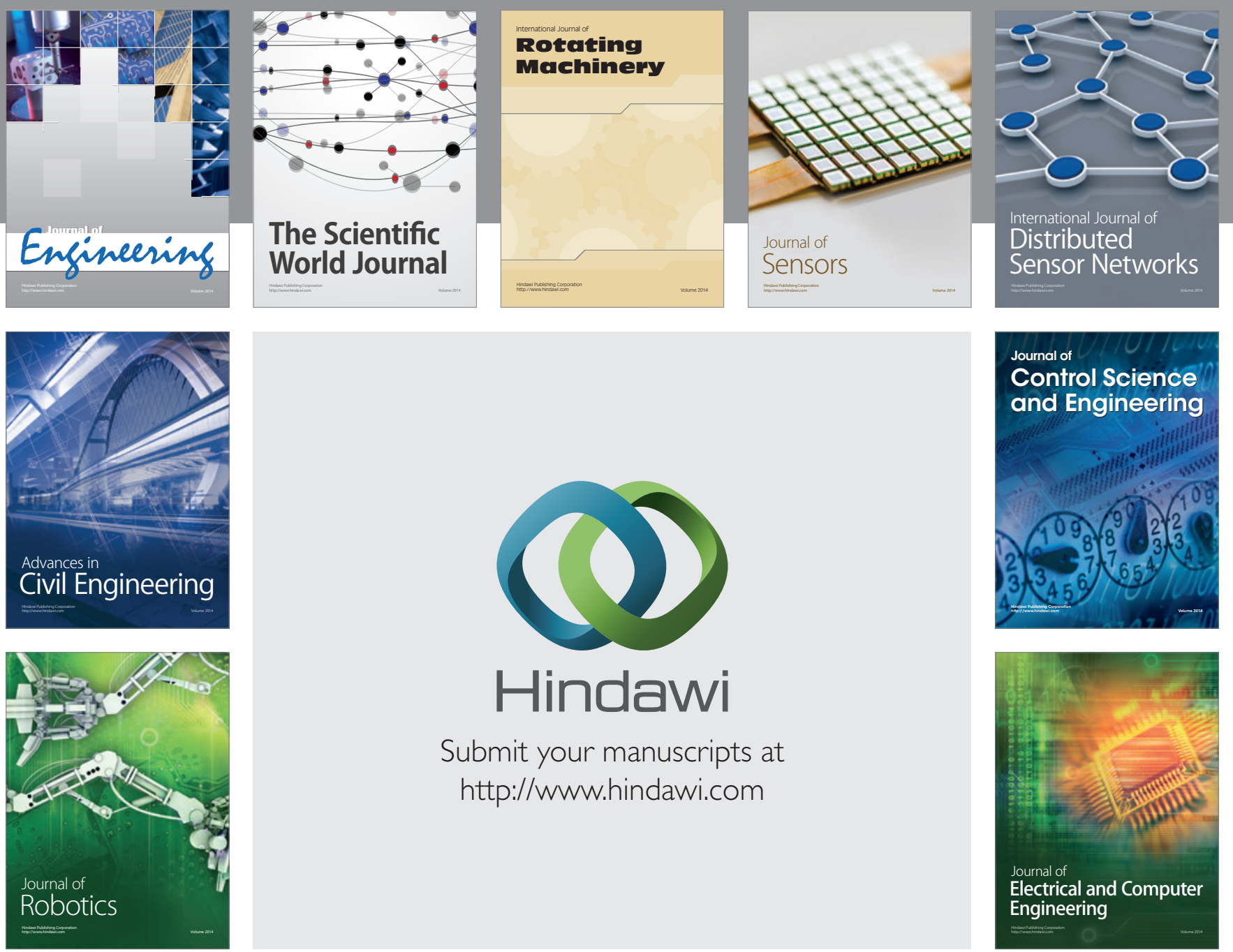

Submit your manuscripts at

http://www.hindawi.com
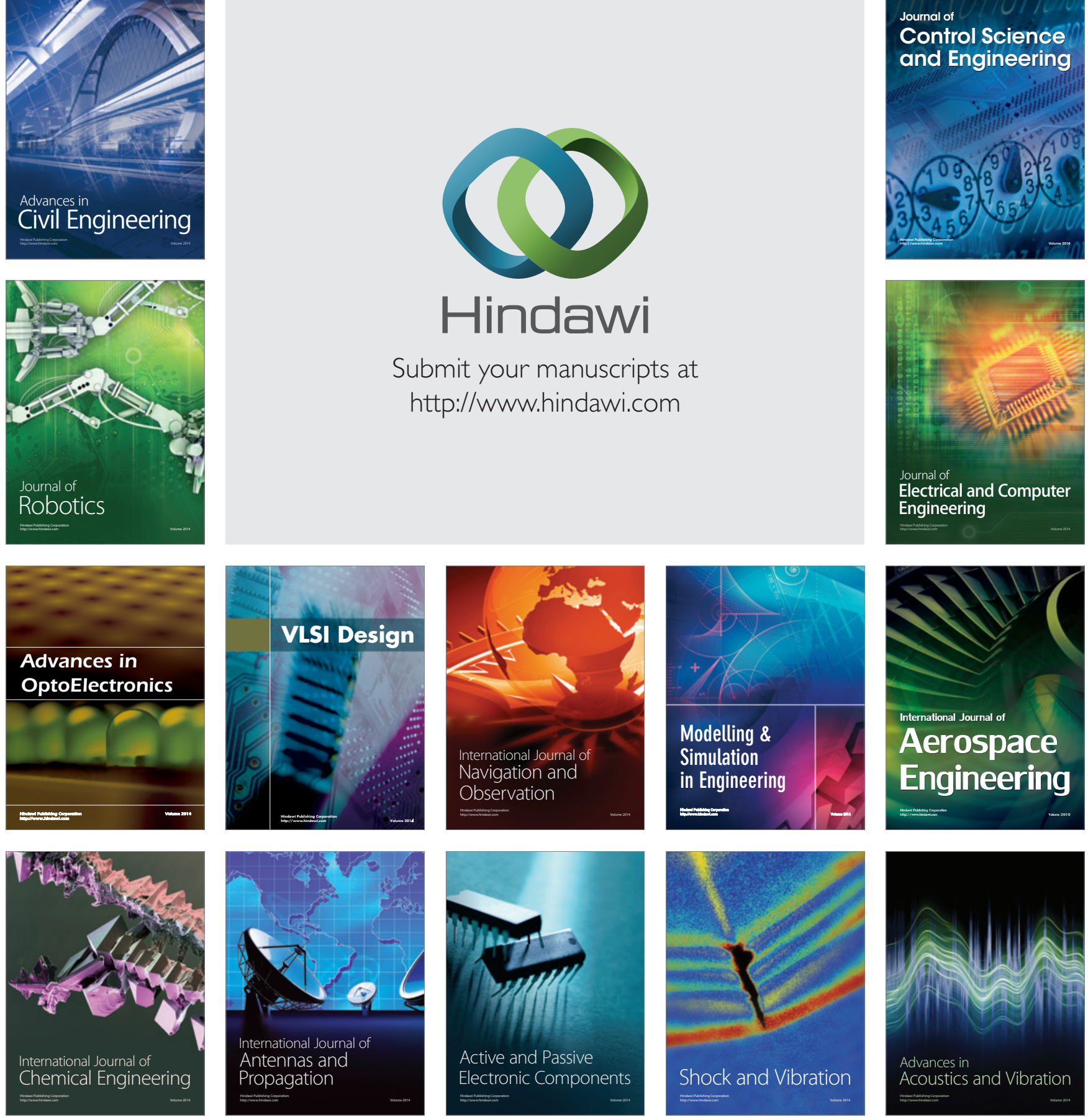